TRANSACTIONS OF THE

AMERICAN MATHEMATICAL SOCIETY

Volume 349, Number 8, August 1997, Pages 3401-3408

S 0002-9947(97)01975-2

\title{
MATRIX EXTENSIONS AND EIGENVALUE COMPLETIONS, THE GENERIC CASE
}

\author{
WILLIAM HELTON, JOACHIM ROSENTHAL, AND XIAOCHANG WANG
}

\begin{abstract}
In this paper we provide new necessary and sufficient conditions for the so-called eigenvalue completion problem.
\end{abstract}

\section{INTRODUCTION}

Let $\operatorname{Mat}_{n \times n}(\mathbb{C})$ be the set of all $n \times n$ matrices having complex entries. In the sequel we will identify $M_{n \times n}$ with the vector space $\mathbb{C}^{n^{2}}$. Let $A \in \operatorname{Mat}_{n \times n}$ be a particular element and let $\mathcal{L} \subset M a t_{n \times n}$ be a complex linear subspace. Identify the set of monic polynomials of degree $n$ in $\mathbb{C}[n]$ with the vector space $\mathbb{C}^{n}$. In this paper we present new conditions which guarantee that the characteristic map

$$
\chi_{A}: \mathcal{L} \longrightarrow \mathbb{C}^{n}, \quad L \longmapsto \operatorname{det}(s I-A-L)=s^{n}-\sigma_{1} s^{n-1}+\cdots+(-1)^{n} \sigma_{n}
$$

is generically surjective, i.e. we will give conditions which guarantee that the image of $\chi_{A}$ contains a nontrivial Zariski open and therefore dense subset. (Recall that a set in $\mathbb{C}^{n}$ is called Zariski open if its complement is the set of zeros of some polynomials).

First we would like to remark that there are two obvious necessary conditions:

1. $\chi_{A}$ is almost onto only if $\operatorname{dim} \mathcal{L} \geq n$.

2. There must be at least one element $L \in \mathcal{L}$ whose $\operatorname{trace} \operatorname{tr}(L) \neq 0$, i.e. $\mathcal{L} \not \subset s l_{n}$.

The main result of this paper states that if both those necessary conditions are satisfied then for a generic set of matrices in $M_{a t}{ }_{n \times n}$ the characteristic map $\chi$ is generically surjective.

There exists a large literature about so-called matrix completion problems, matrix extension problems and inverse eigenvalue problems in different areas of mathematics. We only mention the linear algebra literature [6], [12], [13], [15], the operator theory literature [1], [8], and the control literature [2], [3], [10].

For a treatment of many of these topics we recommend the recent book by Gohberg, Kaashoek and van Schagen [7], which concentrates to a large extent on eigenvalue completion problems. We now indicate the type of results which we consider interesting in connection with this paper.

As it turns out, our main result immediately implies classical theorems in a wide range of situations. We now list some of these.

Received by the editors September 8, 1995 and, in revised form, March 14, 1996.

1991 Mathematics Subject Classification. Primary 15A18; Secondary $93 B 60$.

Key words and phrases. Eigenvalue completion, dominant morphism theorem, inverse eigenvalue problems.

J. Rosenthal is supported in part by NSF grant DMS-9400965, and X. Wang is supported in part by NSF grant DMS-9500594. 
In the linear algebra literature probably one of the earliest results is due to Farahat and Ledermann [5]. The result states that for a matrix whose $(n-1) \times$ $(n-1)$ top left-hand corner is non-derogatory, every characteristic polynomial can be achieved through the choice of entries in the last row and last column.

In 1977 S. Friedland derived an interesting result involving 'diagonal perturbations':

Theorem 1.1. Let $A \in M_{n \times n}$ be arbitrary and let $\mathcal{L}=\mathcal{D}_{n}$ be the set of diagonal matrices. Then $\chi_{A}$ is surjective of mapping degree $n$ !, i.e. when counted with multiplicity

$$
\#\left\{\chi_{A}^{-1}(D)\right\}=n ! \quad \forall D \in \mathcal{D}_{n} .
$$

Both the result of Farahat and Ledermann and that of Friedland belong to the class of matrix completion problems, i.e. one assumes that certain elements in a matrix are fixed and other elements can be freely chosen.

For the general problem at hand probably the strongest result is due to Byrnes and Wang [4], [14]. It covers the situation when $\mathcal{L} \subset M_{a \times t_{n \times n}}$ is a Lie subalgebra:

Theorem 1.2. Given a Lie algebra $\mathcal{L} \subset g l_{n}$. Then $\chi_{A}$ is onto for all $A$ if and only if rank $\mathcal{L}=n$ and some element of $\mathcal{L}$ has distinct eigenvalues.

This result is basically saying that $\chi_{A}$ is onto for all $A$ if and only if $\mathcal{D}_{n} \subset \mathcal{L}$. So the next natural question would be: When is $\chi_{A}$ onto (or almost onto) for a generic matrix $A$ ? That is the motivation for this paper.

In the control literature our theorem covers a wide range of so-called pole placement problems, and we would like to mention only the most prominent of them, namely, the static output pole placement problem. For this one considers a time invariant system

$$
\dot{x}=A x+B u, \quad y=C x, \quad A \in \operatorname{Mat}_{n \times n}, B \in \operatorname{Mat}_{n \times m}, C \in \text { Mat }_{p \times n} .
$$

It is the goal to construct for every monic polynomial $\phi \in \mathbb{C}[s]$ a so-called 'feedback compensator' $u=F y, F \in M a t_{m \times p}$, such that the 'closed loop characteristic polynomial'

$$
\operatorname{det}(s I-A-B F C)=\phi .
$$

Note that in this situation $\mathcal{L}=\left\{B F C \mid F \in M a t_{m \times p}\right\}$ is a subspace (even a Lie subalgebra) of $M_{a} t_{n \times n}$ of dimension at most $m p$. The main result of Brockett and Byrnes [2] states:

Theorem 1.3. If $m p=n$, then for a generic set of matrices $(A, B, C)$ the map

$$
\chi_{A}: \mathbb{C}^{m p} \longrightarrow \mathbb{C}^{n}, \quad F \longmapsto \operatorname{det}(s I-A-B F C)
$$

is surjective, and there are

$$
d(m, p)=\operatorname{deg} \operatorname{Grass}(m, m+p)=\frac{1 ! 2 ! \cdots(p-1) !(m p) !}{m !(m+1) ! \cdots(m+p-1) !}
$$

solutions for each characteristic polynomial.

For a more extensive treatment of the class of inverse eigenvalue problems appearing in the control literature we refer to the survey article by Byrnes [3].

As a word of caution, note that since the dominant morphism theorem (see Lemma 2.1) is not true over $\mathbb{R}$, this paper does not address classical completion 
problems which involve real subspaces $\mathcal{L}$ of $\operatorname{Mat}_{n \times n}(\mathbb{R})$. For example, completion to self-adjoint matrices is not covered here.

In the next section we will provide the main results of this paper. In Section 3 we will explain in geometric terms some of the main ingredients of the proof of Theorem 2.4, the major result of this article.

\section{DOMINANT MORPHISM THEOREM}

AND THE LINEARIZATION OF THE CHARACTERISTIC MAP

Consider once more the characteristic map (1.1):

$$
\chi_{A}: \mathcal{L} \longrightarrow \mathbb{C}^{n}, \quad L \longmapsto\left(-\sigma_{1}(A+L), \ldots,(-1)^{n} \sigma_{n}(A+L)\right),
$$

where $\sigma_{i}(A+L)$ denotes the $i$ th elementary symmetric function of the eigenvalues $\lambda_{1}, \ldots, \lambda_{n}$ of $A+L$. There are classical formulas which express the elementary symmetric functions $\sigma_{i}(A+L)$ uniquely as a polynomial in the power sum symmetric functions

$$
p_{i}:=\lambda_{1}^{i}+\cdots+\lambda_{n}^{i}=\operatorname{tr}(A+L)^{i} .
$$

To be precise, one has the formula (see e.g. [11])

$$
\sigma_{i}(A+L)=\frac{1}{n !} \operatorname{det}\left(\begin{array}{ccccc}
p_{1} & 1 & 0 & \ldots & 0 \\
p_{2} & p_{1} & 2 & & \vdots \\
\vdots & \ddots & \ddots & \ddots & \vdots \\
\vdots & & \ddots & p_{1} & n-1 \\
p_{n} & \ldots & \ldots & p_{2} & p_{1}
\end{array}\right),
$$

which induces an isomorphism $\mathbb{C}^{n} \rightarrow \mathbb{C}^{n},\left(p_{1}, \ldots, p_{n}\right) \mapsto\left(\sigma_{1}, \ldots, \sigma_{n}\right)$. Based on this, we can equally well study the map

$$
\psi: \mathcal{L} \longrightarrow \mathbb{C}^{n}, \quad L \longmapsto\left(\operatorname{tr}(A+L), \ldots, \operatorname{tr}(A+L)^{n}\right),
$$

which we call the trace map for $A$. The main ingredient of our proof will be a linearization of $\psi$. For this we compute the difference quotient

$$
\lim _{\epsilon \mapsto 0} \frac{\operatorname{tr}(A+\epsilon L)^{i}-\operatorname{tr} A^{i}}{\epsilon}=i \cdot \operatorname{tr}\left(A^{i-1} L\right) .
$$

The linearization $d \psi_{0}$ around the origin is therefore given through

$$
d \psi_{0}(L)=\left(\operatorname{tr}(L), 2 \operatorname{tr}(A L) \ldots, n \cdot \operatorname{tr}\left(A^{n-1} L\right)\right) .
$$

Lemma 2.1. If for some $A \in M a t_{n \times n}$ and some linear subspace $\mathcal{L} \subset M a t_{n \times n}$ the linearization $d \psi_{0}$ of the trace map of $A$ is onto, then $\chi_{A}$ is generically surjective, i.e. $\operatorname{Im}\left(\chi_{A}\right)$ contains a Zariski open subset of $\mathbb{C}^{n}$.

Proof. Direct consequence of the dominant morphism theorem (see e.g. [9]).

In the sequel we will assume that $\operatorname{dim} \mathcal{L}=d \geq n$, and we will identify the set of all $d$-dimensional subspaces $\mathcal{L} \subset M a t_{n \times n}$ with the Grassmann variety $\operatorname{Grass}\left(d, \mathbb{C}^{n^{2}}\right)$. Note that $\operatorname{Grass}\left(d, \mathbb{C}^{n^{2}}\right)$ is an irreducible variety, and a subset $U \subset \operatorname{Grass}\left(d, \mathbb{C}^{n^{2}}\right)$ is a generic set if $U$ contains a non-empty Zariski open subset of $\operatorname{Grass}\left(d, \mathbb{C}^{n^{2}}\right)$. With Lemma 2.1 we have: 
Lemma 2.2. If $d \geq n$, then for a generic subset of pairs

$$
(A, \mathcal{L}) \in \mathbb{C}^{n^{2}} \times \operatorname{Grass}\left(d, \mathbb{C}^{n^{2}}\right)
$$

$\chi_{A}$ is almost onto.

Proof. The set of pairs $(A, \mathcal{L})$ whose linearization $d \psi_{0}$ fails to be surjective is an algebraic subset in $\mathbb{C}^{n^{2}} \times \operatorname{Grass}\left(d, \mathbb{C}^{n^{2}}\right)$. Since the complement is certainly nonempty, the result readily follows.

In everyday language the lemma states that for almost all matrices and almost all linear subspaces $\mathcal{L} \in \operatorname{Grass}\left(d, \mathbb{C}^{n^{2}}\right)$ almost all closed characteristic polynomials can be achieved. The following two theorems strengthen this result.

Theorem 2.3. Let $A \in M a t_{n \times n}$ be an arbitrary matrix. Then for a generic set of subspaces in $\operatorname{Grass}\left(n, \mathbb{C}^{n^{2}}\right), \chi_{A}$ is almost onto.

Proof. The set of subspaces $\mathcal{L}$ having the property that $d \psi_{0}: \mathbb{C}^{n} \rightarrow \mathbb{C}^{n}$ is not surjective is an algebraic subset of $\operatorname{Grass}\left(n, \mathbb{C}^{n^{2}}\right)$. By Friedland's result (Theorem 1.1) the result follows.

The main result of the paper is now as follows:

Theorem 2.4. Let $\mathcal{L} \subset M a t_{n \times n}$ be a linear subspace satisfying $\operatorname{dim} \mathcal{L} \geq n$ and $\mathcal{L} \not \subset s l_{n}$. Then for a generic set of matrices $A \in M a t_{n \times n}$ the characteristic map $\chi_{A}$ is almost onto.

The proof of this theorem is not trivial and will require the rest of this section. In order to facilitate the reasoning we will divide the proof into several lemmas.

Let $\pi: M_{n \times n} \longrightarrow \mathbb{C}^{n}$ be the projection onto the diagonal elements. Let $s l_{n} \subset \mathrm{Mat}_{n \times n}$ be the matrices having trace equal to zero and let $V:=\pi\left(s l_{n}\right)$ be the hyperplane defined by

$$
V=\left\{\left(x_{1}, \ldots, x_{n}\right) \in \mathbb{C}^{n} \mid \sum_{i=1}^{n} x_{i}=0\right\} .
$$

If $\left\{e_{1}, \ldots, e_{n}\right\}$ is the standard basis of $\mathbb{C}^{n}$, then $V$ has a basis consisting of the vectors $\left\{e_{1}-e_{2}, \ldots, e_{n-1}-e_{n}\right\}$.

Let $L \in s l_{n}$ be an arbitrary nonzero matrix, and consider the associated polynomial map

$$
\varphi^{L}: G l_{n} \longrightarrow V, \quad S \longmapsto \pi\left(S L S^{-1}\right) .
$$

One readily verifies that the Jacobian around the identity is given through

$$
d \varphi_{I}^{L}: \operatorname{Mat}_{n \times n} \longrightarrow V, \quad X \longmapsto \pi([X, L])=\pi(X L-L X) .
$$

In the sequel we will derive an algebraic criterion which guarantees that the Jacobian $d \varphi_{I}^{L}$ is surjective. For this we will associate to a matrix $L=\left(l_{i j}\right)$ a graph $\mathcal{G}(L)$ which consists of $n$ vertices $v_{1}, \ldots, v_{n}$ with edges between the $i$ th and $j$ th vertex whenever either $l_{i j}$ or $l_{j i}$ is nonzero. In terms of the matrix $L$ we can identify the vertex $v_{i}$ with the $i$ th diagonal element, with the obvious meaning for the edges.

Recall that a graph with $n$ vertices is called connected provided any two vertices can be joined by some path in the graph. If $\mathcal{G}$ is not connected, then there exist a permutation $\sigma$ of $n$ elements and $r$ integers $1 \leq i_{1}<\cdots<i_{r} \leq n$ with the property 
that $v_{\sigma(1)}, \ldots, v_{\sigma\left(i_{1}\right)}$ represents the first connected component, $v_{\sigma\left(i_{1}+1\right)}, \ldots, v_{\sigma\left(i_{2}\right)}$ represents the second connected component and so on.

Corresponding to the permutation $\sigma$ there is a permutation matrix $P_{\sigma}$ having the property that $P_{\sigma} L P_{\sigma}^{-1}$ is a block diagonal matrix $\operatorname{diag}\left(L_{1}, \ldots, L_{r}\right)$ whose $i$ th block $L_{i}$ has an associated connected graph $\mathcal{G}\left(L_{i}\right)$.

Lemma 2.5. For any $L \in M a t_{n \times n}$ the Jacobian $d \varphi_{I}^{L}$ is surjective if and only if the associated graph $\mathcal{G}(L)$ is connected.

Proof. First assume that $\mathcal{G}(L)$ is not connected. Following the remark before the lemma, there is a permutation matrix $P$ such that

$$
\tilde{L}:=P L P^{-1}=\left(\begin{array}{cc}
L_{1} & 0 \\
0 & L_{2}
\end{array}\right)
$$

is block diagonal, where we assume that $L_{1}$ is a $k \times k$ matrix with $1 \leq k<n$.

Clearly the sum of the first $k$ diagonal elements of the matrix $(X \tilde{L}-\tilde{L} X)$ is always zero. It follows that $d \varphi_{I}^{\tilde{L}}$ and therefore also $d \varphi_{I}^{L}$ are both not surjective.

In order to prove the other direction, assume that $\mathcal{G}(L)$ is connected. Let $E_{i j} \in$ $M_{a} t_{n \times n}$ be the matrix whose $i j$ th entry is 1 , and all the other entries are zero. Then

$$
\pi\left(E_{i j} L-L E_{i j}\right)= \begin{cases}l_{j i}\left(e_{i}-e_{j}\right) & \text { if } i \neq j, \\ 0 & \text { if } i=j,\end{cases}
$$

where $\left\{e_{1}, \ldots, e_{n}\right\}$ is the standard basis of $\mathbb{C}^{n}$ as introduced earlier. Since the vertex $v_{i}$ is connected with the vertex $v_{i+1}$ for $i=1, \ldots, n-1$, it follows that there is a path

$$
v_{i}=v_{i_{1}} \leftrightarrow v_{i_{2}} \leftrightarrow \cdots \leftrightarrow v_{i_{s}}=v_{i+1}
$$

connecting the vertices $v_{i}$ and $v_{i+1}$. From the identity (2.2) it follows that the vectors $e_{i_{1}}-e_{i_{2}}, e_{i_{2}}-e_{i_{3}}, \ldots, e_{i_{s-1}}-e_{i_{s}}$ are all in the image of $d \varphi_{I}^{L}$. In particular the vector $e_{i}-e_{i+1}$ is also in the image of $d \varphi_{I}^{L}$. This completes the proof.

Lemma 2.6. Let $L \in M a t_{n \times n}$ be a nonzero matrix having the property that $\pi(L)=$ 0 . Then there exists a matrix $X$ such that for all $\epsilon \neq 0$ the matrix

$$
\hat{L}(\epsilon):=\left(I_{n}+\epsilon X\right) L\left(I_{n}+\epsilon X\right)^{-1}
$$

has the properties:

1. $d \varphi_{I}^{\hat{L}(\epsilon)}\left(\operatorname{Mat}_{n \times n}\right)=V$

2. $\pi(\hat{L}(\epsilon))=0$.

Proof. If the graph of $L$ is connected the result is trivially fulfilled by using $X=0$. If $L$ is not connected there exists a permutation matrix $P$ such that

$$
\tilde{L}:=P L P^{-1}=\left(\begin{array}{cc}
L_{1} & 0 \\
0 & L_{2}
\end{array}\right)
$$

has the properties that the entry $\tilde{l}_{2,1}$ of $\tilde{L}$ is nonzero and the graph of the $k \times k$ submatrix $L_{1}$ is connected. Let $\tilde{X}:=E_{1,(k+1)}+\cdots+E_{1, n}$. Then

$$
I+\epsilon \tilde{X}=\left(\begin{array}{cc}
I_{k} & \epsilon B \\
0 & I_{n-k}
\end{array}\right),
$$

where the first row of $B$ is $(1, \ldots, 1)$ and all the other rows are zero. 
From the identity

$$
\left(I_{n}+\epsilon \tilde{X}\right) \tilde{L}\left(I_{n}+\epsilon \tilde{X}\right)^{-1}=\left(I_{n}+\epsilon \tilde{X}\right) \tilde{L}\left(I_{n}-\epsilon \tilde{X}\right)=\left(\begin{array}{cc}
L_{1} & \epsilon\left(B L_{2}-L_{1} B\right) \\
0 & L_{2}
\end{array}\right)
$$

it follows that for all $\epsilon$ one has

$$
\begin{aligned}
0=\pi(\tilde{L}) & =\pi\left(\left(I_{n}+\epsilon \tilde{X}\right) \tilde{L}\left(I_{n}+\epsilon \tilde{X}\right)^{-1}\right) \\
& =\pi\left(\left(I_{n}+\epsilon P^{-1} \tilde{X} P\right) L\left(I_{n}+\epsilon P^{-1} \tilde{X} P\right)^{-1}\right) .
\end{aligned}
$$

Let $X:=P^{-1} \tilde{X} P$. By the last expression we have that $\pi(\hat{L}(\epsilon))=\pi(L)$, and since the second row of $B L_{2}-L_{1} B$ is $\tilde{l}_{2,1}(1, \ldots, 1)$, the graph of $\hat{L}(\epsilon)$ is connected for all $\epsilon \neq 0$, i.e. by Lemma 2.5 ,

$$
d \varphi_{I}^{\hat{L}(\epsilon)}\left(M_{n \times n}\right)=V
$$

for all $\epsilon \neq 0$.

Remark 2.7. It can be shown easily that the same is true for any $L \neq \lambda I$, but we will not need this result.

Lemma 2.8. Let $\mathcal{L} \subset M a t_{n \times n}$ be a linear subspace of dimension $n, \mathcal{L} \not \subset s l_{n}$. (I.e., $\mathcal{L}$ contains an element with nonzero trace.) Then there exists an $S \in G l_{n}$ such that $\left.\pi\right|_{S \mathcal{L} S^{-1}}$ is one-one, i.e. the projection of $S \mathcal{L} S^{-1}$ onto the diagonal elements is one to one and onto.

Proof. Let $\left\{L_{1}, L_{2}, \ldots, L_{n}\right\}$ be a basis of $\mathcal{L}$ having the property that $L_{1} \notin s l_{n}, L_{i} \in$ $s l_{n}$ for $i=2,3, \ldots, n$. Furthermore we will assume that $\pi\left(L_{1}\right), \ldots, \pi\left(L_{k}\right)$ are linearly independent. If $k<n$ and if $\pi\left(L_{k+1}\right)$ depends linearly on $\pi\left(L_{1}\right), \ldots, \pi\left(L_{k}\right)$, we will show the existence of some $S \in G l_{n}$ such that

$$
\pi\left(S L_{1} S^{-1}\right), \ldots, \pi\left(S L_{k} S^{-1}\right), \pi\left(S L_{k+1} S^{-1}\right)
$$

are linearly independent. The proof of the theorem will then follow from this claim by induction over $k$.

By assumption there are numbers $a_{1}, \ldots, a_{k}$ having the property that

$$
\pi\left(L_{k+1}\right)=a_{1} \pi\left(L_{1}\right)+\cdots+a_{k} \pi\left(L_{k}\right) .
$$

By possibly replacing $L_{k+1}$ through $L_{k+1}-a_{1} L_{1}-\cdots a_{k} L_{k}$ we can assume that $\pi\left(L_{k+1}\right)=0$. Since $L_{k+1}$ is not a diagonal matrix, it follows from Lemma 2.6 that there exist a matrix $X_{1}$ and a number $\epsilon_{1}$ such that the matrices

$$
\tilde{L}_{i}:=\left(I_{n}+\epsilon_{1} X_{1}\right) L_{i}\left(I_{n}+\epsilon_{1} X_{1}\right)^{-1}, i=1, \ldots, k+1
$$

have the properties that $\pi\left(\tilde{L}_{1}\right), \ldots, \pi\left(\tilde{L}_{k}\right)$ are linearly independent, $\pi\left(\tilde{L}_{k+1}\right)=0$, and $d \varphi_{I}^{\tilde{L}_{k+1}}\left(\operatorname{Mat}_{n \times n}\right)=V$. Since $d \varphi_{I}^{\tilde{L}_{k+1}}$ is surjective, there exists a matrix $X$ such that

$$
d \varphi_{I}^{\tilde{L}_{k+1}}(X)=\pi\left(\left[X, \tilde{L}_{k+1}\right]\right) \notin \operatorname{span}\left\{\pi\left(\tilde{L}_{1}\right), \ldots, \pi\left(\tilde{L}_{k}\right)\right\} .
$$

Consider the Taylor series expansions

$$
\pi\left(\left(I_{n}+\epsilon X\right) \tilde{L}_{i}\left(I_{n}+\epsilon X\right)^{-1}\right)=\pi\left(\tilde{L}_{i}\right)+\beta_{i}(\epsilon), \quad i=1, \ldots, k,
$$

and

$$
\pi\left(\left(I_{n}+\epsilon X\right) \tilde{L}_{k+1}\left(I_{n}+\epsilon X\right)^{-1}\right)=\epsilon \pi\left(\left[X, \tilde{L}_{k+1}\right]\right)+\alpha_{k+1}(\epsilon)
$$


where the vectors $\beta_{i}(\epsilon)$ and $\alpha_{k+1}(\epsilon)$ satisfy

$$
\lim _{\epsilon \rightarrow 0} \beta_{i}(\epsilon)=0, \quad i=1, \ldots, k, \quad \text { and } \quad \lim _{\epsilon \rightarrow 0} \frac{1}{\epsilon} \alpha_{k+1}(\epsilon)=0 .
$$

For a sufficiently small $\epsilon>0$,

$$
\left\{\pi\left(\tilde{L}_{1}\right)+\beta_{i}(\epsilon), \ldots, \pi\left(\tilde{L}_{k}\right)+\beta_{i}(\epsilon), \pi\left(\left[X, \tilde{L}_{k+1}\right]\right)+\frac{1}{\epsilon} \alpha_{k+1}(\epsilon)\right\}
$$

are linearly independent, i.e.

$$
\left\{\pi\left(\left(I_{n}+\epsilon X\right) \tilde{L}_{i}\left(I_{n}+\epsilon X\right)^{-1}\right) \mid i=1, \ldots, k+1\right\}
$$

are linearly independent. This completes the induction step and therefore the proof of the lemma.

Proof of Theorem 2.4. Let $\mathcal{L} \subset M a t_{n \times n}$ be given. By possibly restricting to a subspace $\tilde{\mathcal{L}} \subset \mathcal{L}$ we will be able to assume that $\operatorname{dim} \mathcal{L}=n$. The set of matrices $A \in \operatorname{Mat}_{n \times n}$ whose trace map has has surjective linearization $d \psi_{0}$ forms a Zariski open subset of $\mathbb{C}^{n^{2}}$. In order to prove the theorem it is therefore enough to show the existence of one matrix $\hat{A}$ whose trace map has surjective linearization.

By the last lemma there exists a $S \in G l_{n}$ such that $\left.\pi\right|_{S \mathcal{L} S^{-1}}$ is one to one and onto. Let $D$ be the diagonal matrix

$$
D:=\left(\begin{array}{cccc}
1 & & & \\
& 2 & & \\
& & \ddots & \\
& & & n
\end{array}\right)
$$

Then

$$
\mathcal{L} \longrightarrow \mathbb{C}^{n}, \quad L \longmapsto\left(\operatorname{tr}\left(S L S^{-1}\right), \operatorname{tr}\left(D S L S^{-1}\right), \ldots, \operatorname{tr}\left(D^{n-1} S L S^{-1}\right)\right)
$$

is surjective. Let $\hat{A}:=S^{-1} D S$. Since $\operatorname{tr}\left(D^{i} S L S^{-1}\right)=\operatorname{tr}\left(\hat{A}^{i} L\right)$ for $i=0, \ldots, n-1$, it follows that

$$
\mathcal{L} \longrightarrow \mathbb{C}^{n}, \quad L \longmapsto\left(\operatorname{tr}(L), \operatorname{tr}(\hat{A} L), \ldots, \operatorname{tr}\left(\hat{A}^{n-1} L\right)\right)
$$

is surjective. This shows the existence of the desired matrix $\hat{A}$ and completes the proof.

\section{Some GEOMETRIC REMARKS}

The main technical result of this paper is Lemma 2.8. In this section we do some geometric interpretation of this result.

Consider once more the $\operatorname{Grassmannian} \operatorname{Grass}\left(n, \mathbb{C}^{n^{2}}\right)$. The similarity transformation on $\mathrm{Mat}_{n \times n}$ induces a group action

$$
\begin{aligned}
\phi: \quad G l_{n} \times \operatorname{Grass}\left(n, \mathbb{C}^{n^{2}}\right) & \longrightarrow \operatorname{Grass}\left(n, \mathbb{C}^{n^{2}}\right), \\
(S, \mathcal{L}) & \longmapsto S \mathcal{L} S^{-1} .
\end{aligned}
$$

Inside $\operatorname{Grass}\left(n, \mathbb{C}^{n^{2}}\right)$ there are two natural Schubert subvarieties:

$$
\begin{aligned}
& \mathcal{S}_{1}:=\left\{\mathcal{L} \in \operatorname{Grass}\left(n, \mathbb{C}^{n^{2}}\right)|\pi|_{\mathcal{L}} \text { is not surjective }\right\}, \\
& \mathcal{S}_{2}:=\left\{\mathcal{L} \in \operatorname{Grass}\left(n, \mathbb{C}^{n^{2}}\right) \mid \mathcal{L} \subset s l_{n}\right\} .
\end{aligned}
$$

One readily verifies that $\mathcal{S}_{2}$ is isomorphic to a sub-Grassmann variety, $\mathcal{S}_{2} \subset \mathcal{S}_{1}$, and $\mathcal{S}_{1}$ is a Schubert hypersurface, i.e. a codimension one Schubert subvariety. Note 
that $\mathcal{S}_{2}$ is $G l_{n}$ invariant. Lemma 2.8 now states that if $X \in \mathcal{S}_{1}$, then the whole $G l_{n}$ orbit $G l_{n}(X) \subset \mathcal{S}_{1}$ if and only if $X \in \mathcal{S}_{2}$.

Consider now the canonical Hermitian inner product on $\mathbb{C}^{n^{2}} \simeq M a t_{n \times n}$. This inner product induces an isomorphism between $\operatorname{Grass}\left(n, \mathbb{C}^{n^{2}}\right)$ and $\operatorname{Grass}\left(n^{2}-n, \mathbb{C}^{n^{2}}\right)$. Let $\mathcal{S}_{1}^{\perp}$ and $\mathcal{S}_{2}^{\perp}$ be the subvarieties in $\operatorname{Grass}\left(n^{2}-n, \mathbb{C}^{n^{2}}\right)$ corresponding to $\mathcal{S}_{1}, \mathcal{S}_{2}$. One readily verifies that

$$
\begin{aligned}
& \mathcal{S}_{1}^{\perp}:=\left\{\mathcal{W} \in \operatorname{Grass}\left(n^{2}-n, \mathbb{C}^{n^{2}}\right) \mid \mathcal{D}_{n} \cap \mathcal{W} \neq\{0\}\right\} \\
& \mathcal{S}_{2}^{\perp}:=\left\{\mathcal{W} \in \operatorname{Grass}\left(n^{2}-n, \mathbb{C}^{n^{2}}\right) \mid I_{n} \in \mathcal{W}\right\} .
\end{aligned}
$$

Translating Lemma 2.8 into this dual version, we immediately have:

Lemma 3.1. Let $\mathcal{W} \subset M_{n \times n}$ be a linear subspace of dimension $n^{2}-n$ which does not contain the identity matrix $I_{n}$. Then there exists an $S \in G l_{n}$ having the property that $S \mathcal{W} S^{-1}$ contains no diagonal matrix.

\section{REFERENCES}

1. J. A. Ball, I. Gohberg, L. Rodman, and T. Shalom, On the eigenvalues of matrices with given upper triangular part, Integr. Eq. Oper. Th. 13 (1990), 488 - 497. MR 92c: 47015

2. R. W. Brockett and C. I. Byrnes, Multivariable Nyquist criteria, root loci and pole placement: A geometric viewpoint, IEEE Trans. Automat. Control AC-26 (1981), 271-284. MR 82i:93033b

3. C. I. Byrnes, Pole assignment by output feedback, Lecture Notes in Control and Information Sciences \# 135, Springer Verlag, 1989, pp. 31-78. MR 90k:93001

4. C. I. Byrnes and X. Wang, The additive inverse eigenvalue problem for Lie perturbations, SIAM J. Matrix Anal. Appl. 14 (1993), 113 - 117. MR 93m:15023

5. H. K. Farahat and W. Ledermann, Matrices with prescribed characteristic polynomial, Proc. Edinburgh Math. Soc. 11 (1958), 143-146. MR 21:6382

6. S. Friedland, Inverse eigenvalue problems, Linear Algebra Appl. 17 (1977), 15-51. MR $\mathbf{5 7 : 1 2 5 5 0}$

7. I. Gohberg, M. A. Kaashoek, and F. van Schagen, Partially specified matrices and operators: Classification, completion, applications, Birkhäuser, 1995. CMP 97:01

8. I. Gohberg and S. Rubinstein, A classification of upper equivalent matrices: The generic case, Integr. Eq. Oper. Th. 14 (1991), 533 - 543. MR 92f: 15009

9. R. Hartshorne, Algebraic geometry, Springer Verlag, Berlin, 1977. MR 57:3116

10. R. Hermann and C. F. Martin, Applications of algebraic geometry to system theory part I, IEEE Trans. Automat. Control AC-22 (1977), 19-25. MR 56:2530

11. I. G. Macdonald, Symmetric functions and Hall polynomials, Oxford University Press, Oxford, 1979. MR 84g:05003

12. G. N. de Oliveira, Matrices with prescribed characteristic polynomial and principal blocks II, Linear Algebra Appl. 47 (1982), 35-40. MR 84a:15010

13. F. C. Silva, Matrices with prescribed eigenvalues and principal submatrices, Linear Algebra Appl. 92 (1987), 241-250. MR 88h:15019

14. X. Wang, Additive inverse eigenvalue problems and pole placement of linear systems, Ph.D. thesis, Arizona State University, 1989.

15. I. Zaballa, Matrices with prescribed rows and invariant factors, Linear Algebra Appl. 87 (1987), 113-146. MR 88d:15015

Department of Mathematics, Universityof California at San Diego, La Jolla, CaliFORNIA 92093-0112

E-mail address: helton@osiris.ucsd.edu

Department of Mathematics, University of Notre Dame, Notre Dame, Indiana 46556 5683

E-mail address: Rosenthal.1@nd.edu

Department of Mathematics, Texas Tech University, Lubbock, Texas 79409-2013

E-mail address: mdxia@ttacs1.ttu.edu 